\title{
18-cm VLA observations of OH towards the Galactic Centre
}

\section{Absorption and emission in the four ground-state $\mathrm{OH}$ lines ${ }^{\star}$}

\author{
R. Karlsson ${ }^{1}$, L. O. Sjouwerman ${ }^{2,3}$, Aa. Sandqvist ${ }^{1}$, and J. B. Whiteoak ${ }^{4}$ \\ 1 Stockholm Observatory, SCFAB-AlbaNova, 10691 Stockholm, Sweden \\ e-mail: rolandk@astro.su.se \\ 2 Joint Institute for VLBI in Europe, Postbus 2, 7990 AA Dwingeloo, The Netherlands \\ 3 NRAO Array Operations Center, PO Box 0, Socorro NM 87801, USA \\ e-mail: 1sjouwer@aoc.nrao.edu \\ ${ }^{4}$ CSIRO, Australian Telescope National Facility, Box 76, Epping NSW 2121, Australia \\ e-mail: jwhiteoa@atnf.csiro.au
}

Received 8 July 2002 / Accepted 28 February 2003

\begin{abstract}
The OH distribution in the Sgr A Complex has been observed in the 1612-, 1665-, 1667- and 1720-MHz OH transitions with the Very Large Array (VLA) in BnA configuration. Maps are presented with a channel velocity resolution of about $9 \mathrm{~km} \mathrm{~s}^{-1}$ and with angular resolutions of $4^{\prime \prime} \times 3^{\prime \prime}$. Some clear results are highlighted here, such as absorption from the Circumnuclear Disk (CND) and the OH-Streamer inside the CND near Sgr A*, strong absorption towards most of the eastern and western parts of the Sgr A East shell, lack of absorption towards both Sgr A West and the compact HII-regions to the east of Sgr A East, and double-lobed structure of the High Negative Velocity Gas (HNVG) oriented northeast and southwest of Sgr A*, and finally compact, point-like maser emission in all four transitions, in particular a $1720-\mathrm{MHz}$ maser at $-132 \mathrm{~km} \mathrm{~s}^{-1}$ in the CND as counterpart to a $1720-\mathrm{MHz}$ maser at $+132 \mathrm{~km} \mathrm{~s}^{-1}$ in the CND.
\end{abstract}

Key words. Galaxy: center - ISM: individual objects: Sgr A - ISM: molecules - ISM: clouds - masers - surveys

\section{Introduction}

The ${ }^{2} \Pi_{3 / 2}, J=3 / 2$ ground rotational state of the Hydroxyl $(\mathrm{OH})$ radical is split into four hyperfine levels, leading to the well-known 18-cm main line transitions at 1665 and $1667 \mathrm{MHz}$ and satellite line transitions at 1612 and $1720 \mathrm{MHz}$. The relative intensities of the lines expected from an optically thin gas in local thermodynamic equilibrium (LTE) are 1612:1665:1667:1720 = 1:5:9:1. However, the line ratios are highly sensitive to the physical environment. The first highresolution maps of the 18-cm emission from Sgr A and the associated 1665- and 1667-MHz OH molecular gas distribution in the Galactic Centre (GC) region resulted from a series of lunar occultations in 1968 (Sandqvist 1971, 1973, 1974). They revealed that Sgr A consists of at least two components, now known as Sgr A East and Sgr A West (e.g. Downes \& Martin 1971; Ekers et al. 1983), and that the region could be simply modeled by a rotating and contracting cloud of dust and molecules surrounding the continuum source.

\footnotetext{
Send offprint requests to: Aa. Sandqvist, e-mail: aage@astro.su.se

* Figures 1 to 4 are only available in electronic form at http://www. edpsciences.org
}

Since then, interferometers have been playing an increasingly important role in the observations towards the GC. For example, searches for 1612-MHz OH maser emission associated with OH/IR stars (e.g. Lindqvist et al. 1992; Sjouwerman et al. 1998), and detections of individual $1720-\mathrm{MHz} \mathrm{OH}$ masers (Yusef-Zadeh et al. 1996) would not have been possible without interferometers because of confusion (Habing et al. 1983). Furthermore, in order to unravel the complex structure of Sgr A at higher resolution, Whiteoak et al. (1974) and Bieging (1976) used the Owens Valley interferometer to map the 6-cm $\mathrm{H}_{2} \mathrm{CO}$ and $1667-\mathrm{MHz} \mathrm{OH}$ absorption, respectively. Subsequently, the Very Large Array (VLA) has been used in several configurations, with resolutions ranging from about 4" to an arcminute, to study the H I absorption (Liszt et al. 1983; Lasenby et al. 1989; Yusef-Zadeh et al. 1993; Zhao et al. 1995) and the $\mathrm{H}_{2} \mathrm{CO}$ absorption (Whiteoak et al. 1983; Pauls et al. 1996). Magnetic field measurements have been performed using the Zeeman splitting of absorption lines in OH (Killeen et al. 1992) and H I (Plante et al. 1995).

The molecular complex associated with Sgr A has also been mapped in emission lines of many different molecules. Good reviews on the GC and its environment can be found in Morris \& Serabyn (1996) and Mezger et al. (1996). The complex 
Table 1. VLA 18-cm OH observational summary.

\begin{tabular}{ccccc}
\hline \hline $\begin{array}{c}\text { Frequency } \\
(\mathrm{MHz})\end{array}$ & $\begin{array}{c}\mathrm{HPBW} \\
\left({ }^{\prime \prime} \times{ }^{\prime \prime}\right)\end{array}$ & $\begin{array}{c}\text { PA } \\
\left({ }^{\circ}\right)\end{array}$ & $\begin{array}{c}\text { Resolution } \\
\left(\mathrm{km} \mathrm{s}^{-1}\right)\end{array}$ & $\begin{array}{c}t_{\text {integration }} \\
(\mathrm{min})\end{array}$ \\
\hline 1612 & $4.0 \times 2.7$ & 55.3 & 9.1 & 144 \\
1665 & $3.9 \times 2.9$ & 64.7 & 8.8 & 169 \\
1667 & $4.0 \times 2.8$ & 61.1 & 8.8 & 173 \\
1720 & $3.7 \times 2.7$ & 57.1 & 8.5 & 148 \\
\hline
\end{tabular}

consists predominantly of a molecular belt with density concentrations that define the " $+50 \mathrm{~km} \mathrm{~s}^{-1}$ cloud" $(\mathrm{M}-0.02-0.07)$ and the "+20 $\mathrm{km} \mathrm{s}^{-1}$ cloud" (M-0.13-0.08), as well as the Circumnuclear Disk (CND) (see e.g. Sandqvist 1989; Genzel 1989; Zylka et al. 1990; Lindqvist et al. 1995; Coil \& Ho 2000). Other features seen towards the GC are the High Negative Velocity Gas (HNVG) (Yusef-Zadeh et al. 1993) and the Expanding Molecular Ring (EMR) (e.g. Güsten 1989). However, in contrast to emission of the total distribution of molecular gas, absorption only yields the foreground distribution of the molecule with respect to the continuum emission. Some indication of the relative location of the molecular region and the continuum sources can thus be obtained by comparing absorption and emission line data.

In this paper, we present arc-second resolution VLA observations of all four $18-\mathrm{cm} \mathrm{OH}$ lines with the $\mathrm{BnA}$ configuration. As the line intensity ratios of the four $\mathrm{OH}$ lines directly probe the population distribution of the gas, observations of all four lines provide a measure of the physical state of the gas, in particular deviations from thermal equilibrium. We will highlight the most striking results here in Sect. 3, although a detailed physical analysis of the data will be published in a forthcoming paper. Some of the preliminary results have been presented by Sandqvist et al. (1987, 1989).

\section{Observations and data reduction}

The BnA configuration observations were performed in late June 1986 and a summary of these observations is presented in Table 1. The observations were performed with 18 VLA antennas at RHC polarisation using a total bandwidth of $3.125 \mathrm{MHz}$, corresponding to a total velocity coverage of about $550 \mathrm{~km} \mathrm{~s}^{-1}$, divided into 63 spectral channels. The centre line-of-sight velocity with respect to the Local Standard of Rest (LSR) was $-38 \mathrm{~km} \mathrm{~s}^{-1}$ at each of the observed transitions. All references to velocities in this paper refer to line-ofsight velocities with respect to the LSR. We used the NRAO Astronomical Image Processing System (AIPS) for the data reduction. Extensive flagging of corrupted data was needed for the 1612-MHz data set due to interference of the Russian GLONASS satellite system. Standard calibration of the visibility phases was done against the nearby source B1748-253; standard amplitude and bandpass calibration were obtained using 3C 286.

Because the high-velocity tails of the $\mathrm{OH}$ main line transitions (1665 and $1667 \mathrm{MHz}$ ) overlap in the intermediate frequency range (i.e. around $1666 \mathrm{MHz}$ ), a direct subtraction of the continuum in the separate visibility data sets was not possible. Also, even when concatenating together the 1665and $1667-\mathrm{MHz}$ visibility data sets into one continuous frequency band, UVLIN could not be used due to different $(u, v)$-coverage. The continuum was therefore subtracted from the total image cube, made up from the unsubtracted 1665and $1667-\mathrm{MHz}$ image cubes concatenated together. The subtraction was done using IMLIN with a linear fit over featurefree channels on either side of the total $1665-$ and $1667-\mathrm{MHz}$ absorption complex. For self-calibration of the phases we used the continuum image derived with IMLIN, partly because there were no strong masers, and partly because the continuum could not be subtracted from the visibility data. We made maps with $1024 \times 1024$ pixels of 0 ! 75 with both the CLEAN as well as the maximum entropy algorithm (VTESS). Although the two methods gave similar results, the 103-channel image cube was faster to generate with the maximum entropy method (MEM) and of somewhat higher quality, i.e. less mottled. Typical rms noise levels are $5 \mathrm{mJy} /$ beam. The resulting 96 MEM absorption channel maps can be found in Figs. 1 and 2, where some concatenation effects can be seen as noise, e.g. at the left side of the $1667-\mathrm{MHz}$ maps at velocities of +100 to $+140 \mathrm{~km} \mathrm{~s}^{-1}$ in Fig. 2. Note that although Figs. 1 and 2 are labeled with velocities for 1665 and $1667 \mathrm{MHz}$, respectively, Figs. 1 and 2 do form one continuous sequence.

In order to produce the maps of the absorption and line emission in the $\mathrm{OH}$ satellite line transitions (1612 and $1720 \mathrm{MHz}$ ), we subtracted out a linear fit over frequency to the continuum directly from the visibilities with UVLIN. A strong maser spot was then used to self-calibrate both the amplitudes and phases of the visibilities. For the 1612- as well as the $1720-\mathrm{MHz}$ data we made 63 cleaned channel maps of $1024 \times 1024$ pixels of 0. ' 75 with IMAGR. The relevant 48 channels roughly covering $+200 \gtrsim v_{\mathrm{LSR}} \gtrsim-200 \mathrm{~km} \mathrm{~s}^{-1}$ are shown in Figs. 3 and 4. We also checked our CLEAN maps for 1612 and $1720 \mathrm{MHz}$ by using the MEM algorithm for a small number of selected channels. No significant differences were found.

\section{Results}

Below we first discuss results on the $\mathrm{OH}$ absorption measurements; a second section discusses the $\mathrm{OH}$ emission features.

\section{1. $\mathrm{OH}$ absorption results}

The $\mathrm{OH}$ results, presented here in Figs. 1-4, have not been corrected for the effect of the background continuum distribution on the absorption distribution. For the simple case, where all the continuum is behind the absorbing clouds, the distribution of the line-to-continuum is the best indicator of the variation of the $\mathrm{OH}$ column density. This apparent opacity is given by $-T_{\mathrm{L}} / T_{\mathrm{C}}$ where $T_{\mathrm{L}}$ is the absorption line temperature which is a negative number, and $T_{\mathrm{C}}$ is the continuum temperature. Apparent opacity maps have been produced for several features and are presented below. The situation in the Sgr A Complex is, however, considerably more complicated since some of the continuum may be in front of some of the absorbing clouds. Furthermore, there is an additional problem inherent in interferometer observations: if the contiuum distribution is more 
extended than the line absorption feature (which is true for the case of the Sgr A Complex), then the continuum will sometimes be more resolved out leading to incorrect continuum levels. This, in turn, leads to the possibility of $-T_{\mathrm{L}} / T_{\mathrm{C}}$ being greater than one, in some regions. This problem will require a certain amount of modelling and will be studied in more detail in a subsequent paper.

\subsubsection{Molecular belt +50 and $+20 \mathrm{~km} \mathrm{~s}^{-1}$-clouds}

The Sgr A +50 and $+20 \mathrm{~km} \mathrm{~s}^{-1}$-clouds appear to be massive condensations immersed in an extended molecular belt which stretches in a northeastern direction from south of the continuum Sgr A Complex to east of the Complex. While the cores of the two molecular clouds lie just outside the continuum Complex, the outer lower levels of the belt do coincide with the directions to the continuum components. The concept of a continuous belt is further strengthened by the gradual, although not linear, velocity gradient from positive velocities in the northeast through the $+50 \mathrm{~km} \mathrm{~s}^{-1}$ and the $+20 \mathrm{~km} \mathrm{~s}^{-1}$ clouds to negative velocities in the southwest (see e.g. Sandqvist 1989). The line-of-sight location of the different continuum and molecular components have been presented schematically by Coil \& Ho (2000) and earlier by Sandqvist (1989) and Zylka et al. (1990).

It is apparent in all four lines, but especially in the highresolution 1665- and 1667-MHz maps in Figs. 1 and 2, that the gas in the the molecular belt is seen clearly in absorption against the shell structure of Sgr A East - the eastern and most of the western parts of the shell - but not against the spiral structure of Sgr A West. See, for example, the sequence of velocity maps from +67.7 to $+23.6 \mathrm{~km} \mathrm{~s}^{-1}$ in the $1667-\mathrm{MHz}$ line in Fig. 2 (or in colour in Fig. 5; see also the $-T_{\mathrm{L}} / T_{\mathrm{C}}$ maps in Fig. 6) where the absorption can be followed across the major part of the Sgr A East shell. The dominant absorption moves westward as the velocity decreases, in agreement with the well-known velocity gradient which exists along the molecular belt. However, there is an obvious lack of absorption at the velocities around +32.4 and $+23.6 \mathrm{~km} \mathrm{~s}^{-1}$ across Sgr A West and the expected western part of the Sgr A East shell at this position. Contrast this sequence with the $+6.0 \mathrm{~km} \mathrm{~s}^{-1}$ map where at least the North Arm of Sgr A West is seen clearly being absorbed. The absorption in this latter map originates in a widespread overlying cloud well in front of Sgr A and the associated absorption distribution reflects the case where absorption is occurring against all the continuum in each direction. This may imply that a part of the molecular belt lies between the two continuum components, behind Sgr A West and in front of Sgr A East, implying in turn that Sgr A East is behind Sgr A West. Pedlar et al. (1989) and later investigations have presented strong evidence that Sgr A East lies behind Sgr A West. On the other hand, one would in this case still expect to see some absorption towards the Sgr A East western shell in the region of Sgr A West, and none is seen. There may therefore be a real distinct lack of absorbing $\mathrm{OH}$ gas in this region at the expected velocities. This missing gas may have been dispersed by the high velocities if Sgr A East and West are intertwined with the molecular belt. Alternatively, it may have been dissociated by the strong UV radiation field present in this region.

There seems to be very little absorption in the molecular belt towards Sgr A*, shown in the images of Figs. 1-4 as a cross, or in Figs. 5 and 6 as a tiny contour, at position (B1950.0) 17:42:29.33 - 28:59:18.6, but here the picture is complicated by the $\mathrm{OH}-\mathrm{Streamer}$ which is discussed in the next Sect. (3.1.2). Not until the velocity drops to $-2.8 \mathrm{~km} \mathrm{~s}^{-1}$ is there clear unresolved absorption towards Sgr A*, and this can then be followed only through the -11.6 and $-20.4 \mathrm{~km} \mathrm{~s}^{-1}$ maps which are not related to the molecular belt with its velocities of between +20 and $+30 \mathrm{~km} \mathrm{~s}^{-1}$ at the position of Sgr A* We believe that the absorption towards Sgr A* is really this localized and is related in some intriguing manner to the $\mathrm{OH}-\mathrm{Streamer}$. By $-29.2 \mathrm{~km} \mathrm{~s}^{-1}$ the absorption has disappeared and does not appear again until the velocity region covered by the EMR, namely -126.0 to $-143.6 \mathrm{~km} \mathrm{~s}^{-1}$.

It is significant that no absorption at the molecular belt velocities is seen towards the compact H II regions lying just east of the SgrAEast shell, implying that these star formation regions must lie on the near (sunward) side of the molecular belt. Only in the $1667-\mathrm{MHz} \mathrm{OH}$ line at velocities near $-126.0 \mathrm{~km} \mathrm{~s}^{-1}$ is there weak absorption towards these H II regions. This again is the velocity range of the EMR, so, at the very least, they are inside this molecular feature of the GC region. Their positions coincide quite well with the peak of the $+50 \mathrm{~km} \mathrm{~s}^{-1}$ cloud (e.g. Sandqvist 1989), and Goss et al. (1985) have in fact detected $\mathrm{H} 76 \alpha$ recombination lines from the $\mathrm{H}$ II regions with velocities in the range +43 to $+52 \mathrm{~km} \mathrm{~s}^{-1}$. These velocities are somewhat blueshifted with respect to the $+50 \mathrm{~km} \mathrm{~s}^{-1}$ cloud, which would be in agreement with a model indicating their outflowing from the cloud, thus placing them on the near side of the cloud. This is also in agreement with the conclusions drawn by Cotera et al. (2000) from observations of interstellar extinction in the near infrared. Our OH observations would thus seem to support the association of the $\mathrm{H}$ II regions with the near side of the $+50 \mathrm{~km} \mathrm{~s}^{-1}$ cloud.

A perusal of the satellite line maps in Figs. 3 and 4 shows that the maximum $\mathrm{OH}$ absorption towards Sgr A East is considerably deeper in the $1612-\mathrm{MHz}$ line than in the $1720-\mathrm{MHz}$ line, about $60 \mathrm{mJy} / \mathrm{beam}$ versus $45 \mathrm{mJy} / \mathrm{beam}$, respectively. This phenomenon is generally true for most of the absorption seen in these maps.

\subsubsection{The CND and the OH-Streamer}

The Northeast and Southwest $\mathrm{OH}$ components of the CND seen already in the 1968 occultation maps (Sandqvist 1974) are very clear in the VLA BnA maps. They can be traced out to velocities of +150 and $-150 \mathrm{~km} \mathrm{~s}^{-1}$, respectively, in both the 1665- and 1667-MHz lines in Figs. 1 and 2. The signature of the rotating torus is especially clear southwest of Sgr A* in maps at velocities ranging from -20 to $-100 \mathrm{~km} \mathrm{~s}^{-1}$ where the peak absorption intensities reach 89 and $106 \mathrm{mJy} /$ beam for the $1665-$ and $1667-\mathrm{MHz} \mathrm{OH}$ lines, respectively. The northeastern part of the CND can be seen, for example, in the +94.1 to $+120.5 \mathrm{~km} \mathrm{~s}^{-1}$ maps where the peak absorption intensities 
are $68 \mathrm{mJy} / \mathrm{beam}$ for both the 1665- and 1667-MHz lines. While considerably weaker, the CND can still be traced in the 1612-MHz OH line in Fig. 3, and even in the $1720-\mathrm{MHz}$ $\mathrm{OH}$ line in Fig. 4 although only then with some difficulty.

A unique feature - which we called the " $1667-\mathrm{MHz}$ OH Streamer" in our preliminary papers (Sandqvist et al. 1987, and Sandqvist et al. 1989) - is now seen to have been detected in three of the four $\mathrm{OH}$ lines, namely the 1612-, 1665and $1667-\mathrm{MHz}$ lines. It is not detectable in the $1720-\mathrm{MHz}$ line. This molecular gas streamer stretches from the CND's southwestern region inwards through the cavity, inside the CND (at least in projection), to the compact non-thermal radio source, Sgr A*.

The $\mathrm{OH}-\mathrm{Streamer}$ can best be followed in the $1667-\mathrm{MHz}$ maps of Fig. 2. We also present the six most important channel maps $\left(+67.6\right.$ to $+23.6 \mathrm{~km} \mathrm{~s}^{-1}$ ) of Fig. 2 in colour in Fig. 5. It may first be (marginally) distinguished from other features at a velocity of $+76.4 \mathrm{~km} \mathrm{~s}^{-1}$. At a velocity of $+67.6 \mathrm{~km} \mathrm{~s}^{-1}$ it is seen to cut across the southwestern part of the CND and into the cavity. It stands out clearest at a velocity of $+58.8 \mathrm{~km} \mathrm{~s}^{-1}$. Note that the negative velocity of the CND in this region differs from that of the OH-Streamer by more than $100 \mathrm{~km} \mathrm{~s}^{-1}$, and the OH-Streamer may not necessarily be in the same plane as the CND. At these velocities, the "head" of the OH-Streamer (the part closest to Sgr A*) does not coincide with Sgr A* but lies slightly to its northwest. The $\mathrm{OH}-\mathrm{Streamer}$ then "shrinks" and moves closer to $\mathrm{Sgr} \mathrm{A}^{*}$ as the velocity drops until it coincides with $\mathrm{Sgr} \mathrm{A}^{*}$ near $+23.6 \mathrm{~km} \mathrm{~s}^{-1}$. Its coincidence with $\mathrm{Sgr}^{*}$ can then be followed through to the $-20.4 \mathrm{~km} \mathrm{~s}^{-1}$ map, after which there is no more detectable absorption towards Sgr A*.

It is intriguing to follow the structure of the "head" of the $\mathrm{OH}-$ Streamer as its trailing and shrinking part sweeps in a counterclockwise direction while the velocities drop from +50.0 to $-20.4 \mathrm{~km} \mathrm{~s}^{-1}$. From pointing towards the "tail" of the OH-Streamer in a southwest direction at $+50 \mathrm{~km} \mathrm{~s}^{-1}$, the trailing end of the head points west at $+41.2 \mathrm{~km} \mathrm{~s}^{-1}$, north at +32.4 and $+23.6 \mathrm{~km} \mathrm{~s}^{-1}$. This gradual movement inwards and final coincidence with $\operatorname{Sgr~A}^{*}$ makes us believe that the $\mathrm{OH}-$ Streamer does in fact (and not just in projection) exist in the cavity inside the CND and interacts with Sgr A* . An orbital model for the $\mathrm{OH}-\mathrm{Streamer}$ will be developed and presented in a future paper.

We have produced $-T_{\mathrm{L}} / T_{\mathrm{C}}$ maps for the $1667-\mathrm{MHz}$ $\mathrm{OH}$ line in the velocity range +67.7 to $+23.6 \mathrm{~km} \mathrm{~s}^{-1}$ and present them in colour in Fig. 6. Here we have assumed that all the contiuum is behind the $\mathrm{OH}$ gas. The effect of the velocity gradient across the molecular belt, discussed in Sect. 3.1.1, is seen also in the $-T_{\mathrm{L}} / T_{\mathrm{C}}$ maps. The $\mathrm{OH}-\mathrm{Streamer}$ retains its shape and general behaviour although it may be somewhat wider than was apparent in the line intensity maps in Fig. 5. The assumption that the $\mathrm{OH}-\mathrm{Streamer}$ is in front of the southwest arm of Sgr A West may thus be correct. Its $-T_{\mathrm{L}} / T_{\mathrm{C}}$ value is considerably lower, though, than the gas in the molecular belt seen against the shell of Sgr A East.

The OH-Streamer can also be traced in the same velocity maps of the $1665-\mathrm{MHz} \mathrm{OH}$ line in Fig. 1. In the $1612-\mathrm{MHz}$ $\mathrm{OH}$ line maps of Fig. 3, the $\mathrm{OH}$-Streamer is detectable at $+61.9 \mathrm{~km} \mathrm{~s}^{-1}$ and also seen at +71.0 and $+52.8 \mathrm{~km} \mathrm{~s}^{-1}$. We were not able to recognize any absorption by the $\mathrm{OH}-\mathrm{Streamer}$ in the $1720-\mathrm{MHz}$ line (Fig. 4). The relative intensities of the absorptions in the two main lines are not far from those expected (5:9) from an optically thin gas in LTE. In the head of the OH-Streamer, near Sgr A*, the ratio of the 1665:1667-MHz line intensities, $42 \mathrm{mJy} /$ beam:79 mJy/beam, is about 5:9.4. The ratio increases slightly to about 5:7.5 (20 mJy/beam:30 mJy/beam) in the tail which extends southwestward towards the CND, possibly indicating the onset of $1667-\mathrm{MHz}$ line saturation caused by higher density in this region. The $-T_{\mathrm{L}} / T_{\mathrm{C}}$ maps in Fig. 6 also indicate higher densities in the tail than in the head. However, the absorption intensities of the 1612-MHz line are 20 and $12 \mathrm{mJy} /$ beam in the head and tail, respectively, which is surprisingly high. The excitation of the lines is obviously complex and requires deeper study. It is also difficult to understand how the $\mathrm{OH}-$ Streamer can survive in the strong UV radiation field in the cavity inside the CND, but it may be very young and the $\mathrm{OH}$ may be protected to some extent if it is intermingled with dust.

\subsubsection{Other absorption features in the BnA maps}

In Sect. 3.1.1, we briefly mentioned the absorption towards Sgr A* at velocities near -126.0 to $-143.6 \mathrm{~km} \mathrm{~s}^{-1}$ caused by the EMR. At a velocity of $-152.4 \mathrm{~km} \mathrm{~s}^{-1}$ there is practically no $\mathrm{OH}$ absorption, but starting at $-161.3 \mathrm{~km} \mathrm{~s}^{-1}$, reaching a maximum near $-178.9 \mathrm{~km} \mathrm{~s}^{-1}$ and petering out near $-222.9 \mathrm{~km} \mathrm{~s}^{-1}$ there is extended $\mathrm{OH}$ absorption in the region of Sgr A West. This HNVG structure is apparently double-lobed as seen in the $\mathrm{OH}$ gas, one lobe to the northeast of $\mathrm{Sgr} \mathrm{A}^{*}$, the other to the southwest of $\mathrm{Sgr} \mathrm{A}^{*}$, somewhat similar to the orientation of the inner part of the CND. This double-lobed appearance is retained even in $-T_{\mathrm{L}} / T_{\mathrm{C}}$ maps, an example of which is shown in Fig. 7. Only the southwest lobe, though, is seen at the very negative velocities between -196.5 and $-222.9 \mathrm{~km} \mathrm{~s}^{-1}$ (see Fig. 2). When studying a feature in the overlapping velocity range of the 1665 - and $1667-\mathrm{MHz}$ lines, confusion can be mostly eliminated by studying the corresponding feature in the high negative velocity range of the $1667-\mathrm{MHz}$ line and the high positive velocity range of the $1665-\mathrm{MHz}$ line, respectively. The southwest HNVG can also be seen in the -165.6 and $-174.1 \mathrm{~km} \mathrm{~s}^{-1}$ maps of the $1720-\mathrm{MHz} \mathrm{OH}$ line and the northeast part weakly in the $1612-\mathrm{MHz}$ line at $-174.2 \mathrm{~km} \mathrm{~s}^{-1}$. The HNVG, as seen in H I by Yusef-Zadeh et al. (1993) is predominantly distributed along the rotation axis of the CND. A detailed model for the HNVG has been developed by Zhao et al. (1995).

\section{2. $\mathrm{OH}$ emission results}

The four sets of "maxmaps" and "minmaps" of the data cubes are shown in Figs. 8 and 9. The maxmap/minmap presentation method was developed by Sjouwerman (1997). The maxmaps (or minmaps) are produced through projection of the threedimensional data cube onto a two-dimensional sky image map. This is done by storing the maximum (or minimum) intensity over the whole velocity axis for each pixel in the sky 


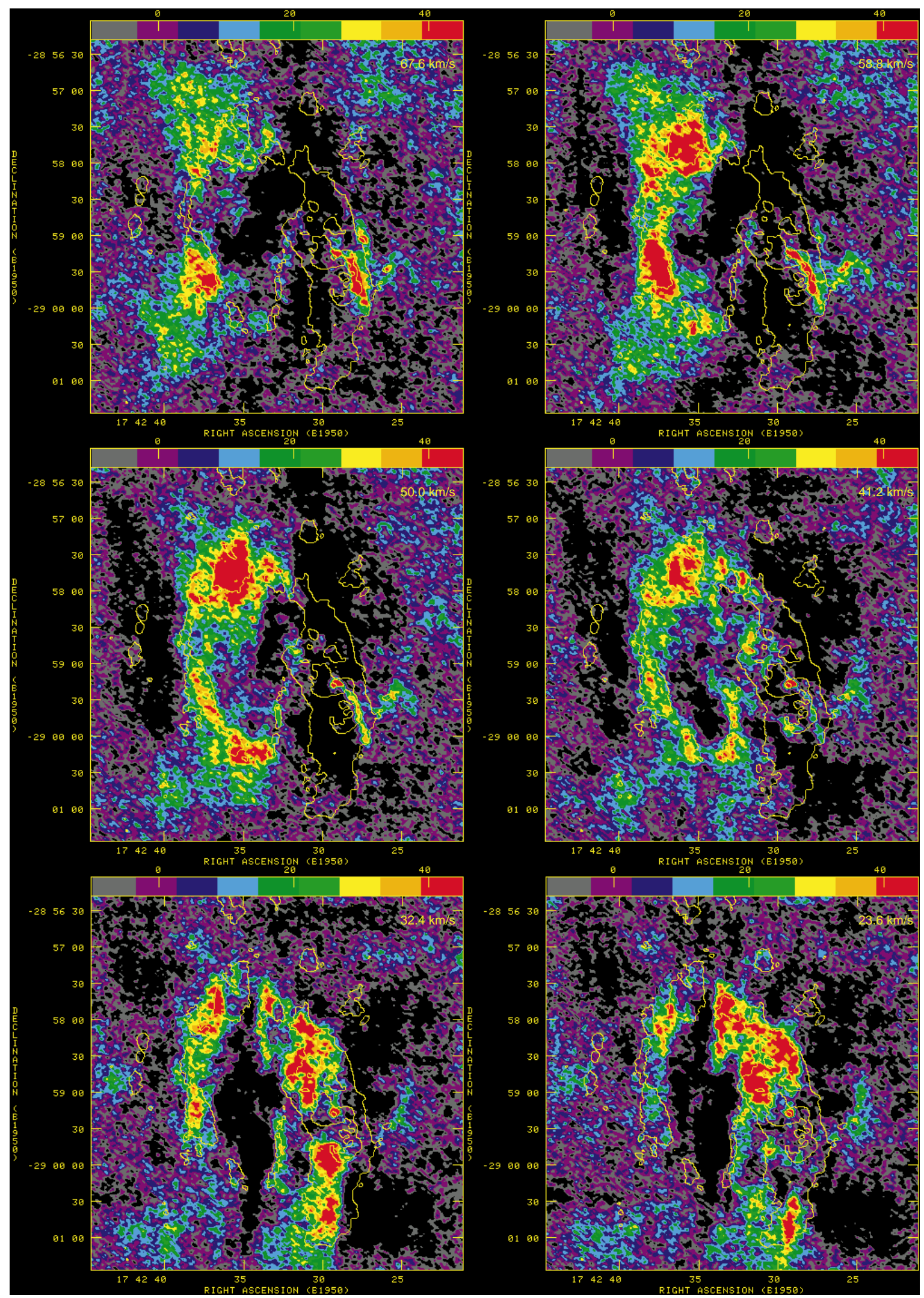

Fig. 5. 1667-MHz OH absorption, highligting in colour the OH-Streamer near Sgr A* and the absorption towards Sgr A East. The range in colour is in $\mathrm{mJy} / \mathrm{beam}$. The velocities are indicated in the upper right corner. The contour level of $15 \mathrm{mJy} / \mathrm{beam}$ indicates Sgr A East and the three compact H II-regions to its east, the contour level 93 mJy/beam indicates Sgr A West, and the contour level $500 \mathrm{mJy} / \mathrm{beam}$ indicates Sgr A*. 

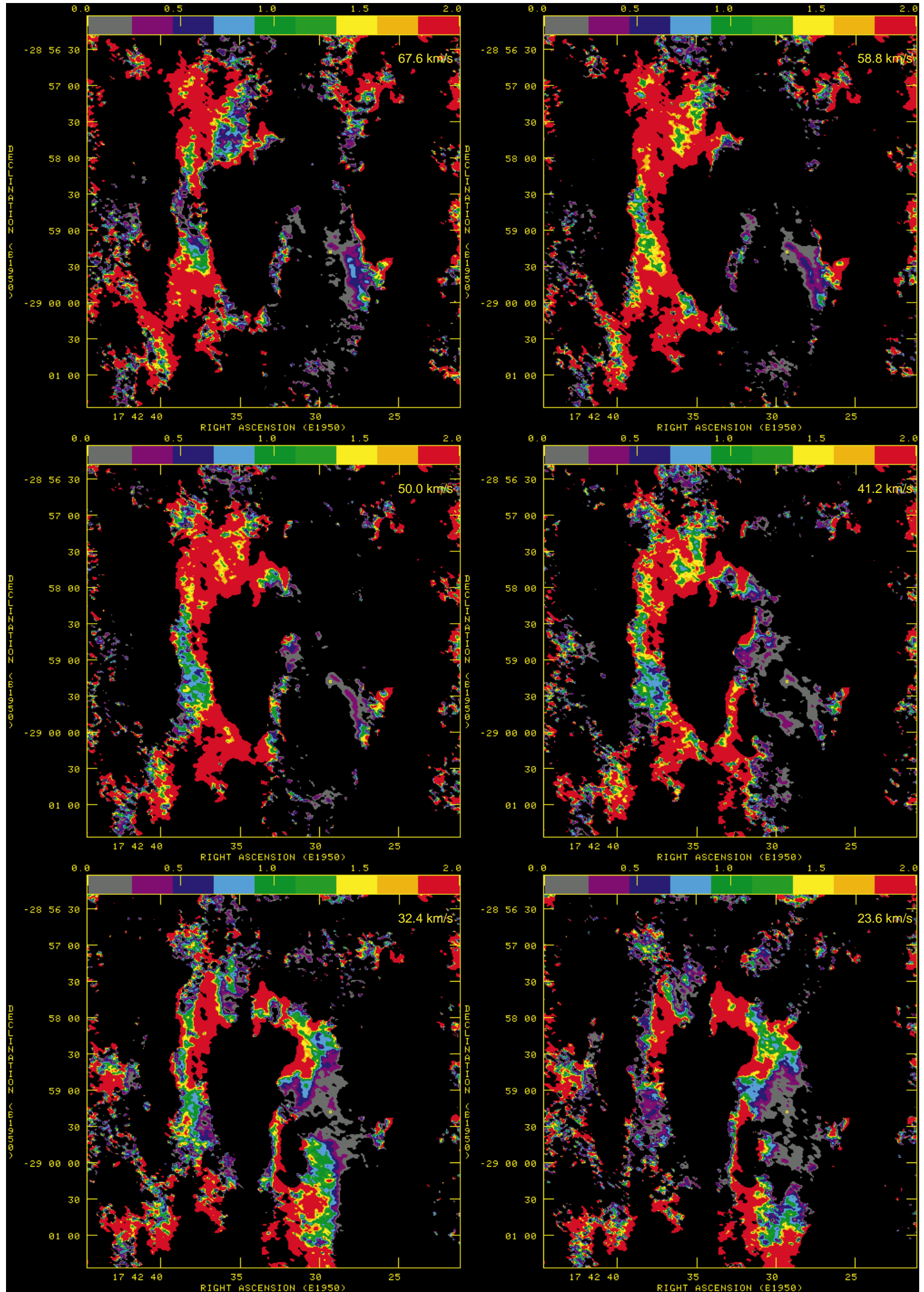

Fig. 6. 1667-MHz OH line-to-continuum distribution $\left(-T_{\mathrm{L}} / T_{\mathrm{C}}\right)$, highligting in colour the OH-Streamer near Sgr $\mathrm{A}^{*}$ and the absorption towards Sgr A East. The range of the colour scale is from 0.0 to 2.0. The velocities are indicated in the upper right corner. The position of Sgr $\mathrm{A}^{*}$ is indicated by a tiny yellow contour. 


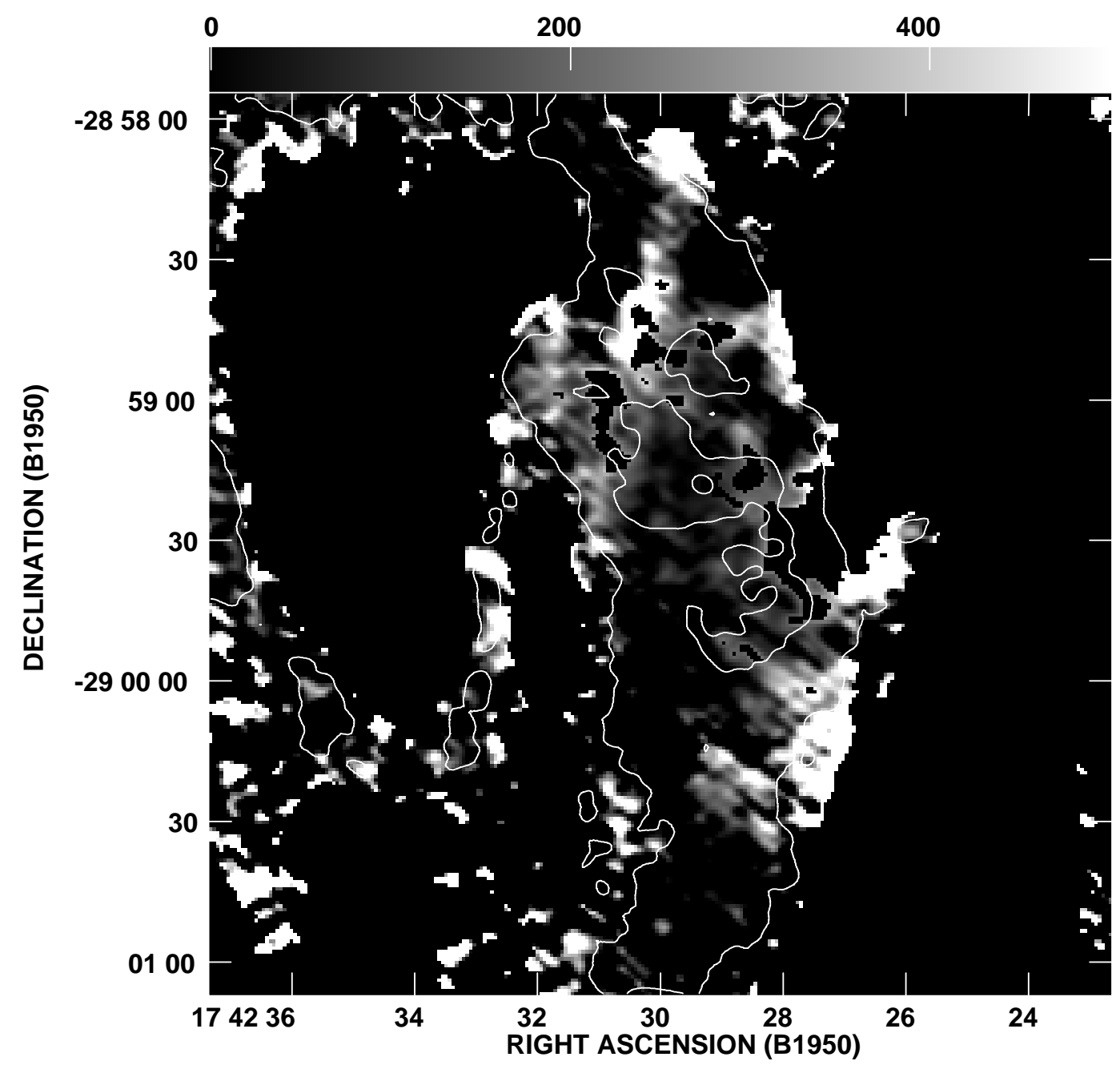

Fig. 7. $1667-\mathrm{MHz} \mathrm{OH}$ line-to-continuum distribution $\left(-T_{\mathrm{L}} / T_{\mathrm{C}}\right)$ for the High Negative Velocity Gas (HNVG) at $v_{\mathrm{LSR}}=-170.1 \mathrm{~km} \mathrm{~s} \mathrm{~s}^{-1}$ The range of the gray scale is from 0 to 0.500 . The contour level of $15 \mathrm{mJy} / \mathrm{beam}$ indicates the western shell of Sgr A East, the contour level $93 \mathrm{mJy} /$ beam indicates Sgr A West, and the contour level $500 \mathrm{mJy} /$ beam indicates Sgr A*

plane. Maxmaps and minmaps can nowadays easily be generated with the AIPS task SQASH. The maxmaps are useful tools for identifying masers and possible regions of extended emission, while the minmaps give an overall view of the spatial distribution of the absorption.

Weak extended emission can be seen in the main line maxmaps in Fig. 8. Although weak, extended and diffuse $\mathrm{OH}$ emission has been reported elsewhere in the Galactic Centre region, in the $1720-\mathrm{MHz}$ satellite line (Yusef-Zadeh et al. 1999), and in other galaxies in the 1667-MHz line (e.g. Pihlström et al. 2001), the extended $\mathrm{OH}$ line emission features in our observations may be indicative of an instrumental effect. We consider it unlikely to have introduced this weak emission by using a non-standard continuum subtraction for the main line $\mathrm{OH}$ data. We do not see any extended emission regions in the satellite lines (Fig. 9), where we have also carefully checked especially individual $1720-\mathrm{MHz}$ maps with velocities close to those of narrow emission features in single-dish integrated spectra (e.g. Whiteoak \& Gardner 1976).

It is known that lack of zero- and short-spacing observations in interferometric data may cause spurious emission or absorption features in interferometer maps. The lack of visibility data from short baselines results in an unsampled central hole in the $(u, v)$-coverage, which cannot be reconstructed correctly by the deconvolution algorithms without a-priori information (such as single-dish observations). In our case, this results in an insensitivity to structures larger than about $80^{\prime \prime}$. This effect may also lead to a (negative) bowl-like structure around strong emission in the central parts of the brightness distribution. The velocity line maps and the background continuum are both affected, although somewhat offset in position from each other depending upon their relative intensity distribution. Ghost line emission features may therefore occur after continuum subtraction. The negative bowl appears to be of the order of $10 \mathrm{mJy} /$ beam, i.e. about $15 \%$ of the extended emission of the Sgr A Complex.

\subsubsection{Point-like $\mathrm{OH}$ masers}

A large number of maser emission point sources are visible in the satellite lines of $1612-$ and $1720-\mathrm{MHz} \mathrm{OH}$ maxmaps in Fig. 9. Some 1665- and 1667-MHz OH main line maser point 


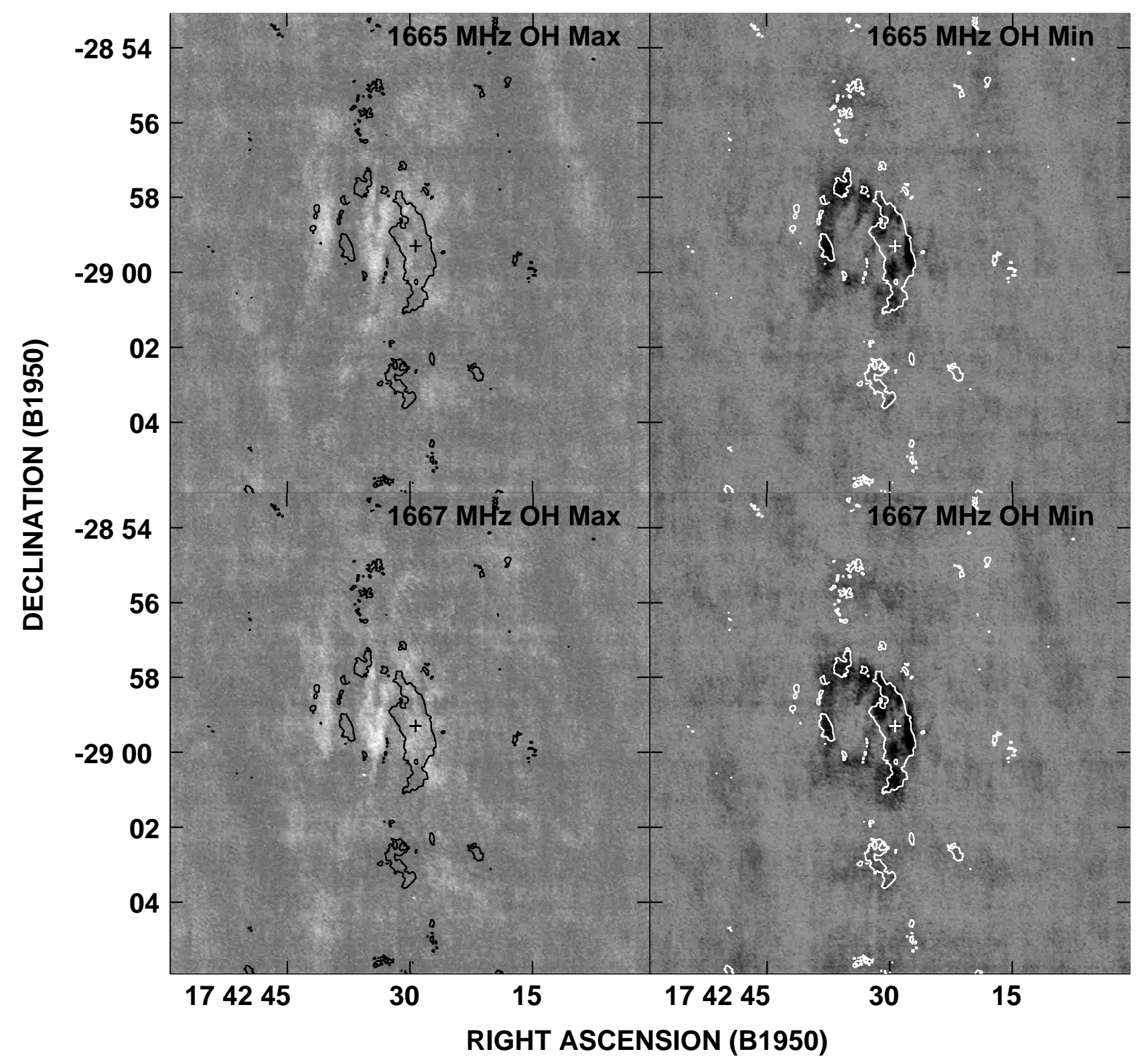

Fig. 8. 18-cm OH main line summary; Maxmaps and Minmaps at $1665 \mathrm{MHz}$ and $1667 \mathrm{MHz}$. Continuum contours and a cross at the position of Sgr A* are placed to guide the eye. The gray scale showing the relative noise of the maps is set to match that of the satellite lines (see text).

sources are also visible in Fig. 8. The entire list of emission point source detections is presented in Table 2. Although these data were taken in 1986, the 1612-MHz maser sources have already been published by Lindqvist et al. (1992; L-92) and subsequently by Sjouwerman et al. (1998; S-98), and most of the 1720-MHz masers by Yusef-Zadeh et al. (1996; YZ96).

There are four new $1720-\mathrm{MHz} \mathrm{OH}$ masers in Table 2, of which the most interesting may be OH359.930-0.049 and which meanwhile also has been noticed in this 1986 data set, and commented on by Yusef-Zadeh et al. (2001; YZ01). Its $v_{\text {system }}$ of $-132 \mathrm{~km} \mathrm{~s}^{-1}$ and its position in the southwestern part of the CND makes it an important symmetric counterpart to the YZ96 maser "B" with its $v_{\text {system }}$ of $+132 \mathrm{~km} \mathrm{~s}^{-1}$ in the northeastern part of the CND. It suggests a mass enclosed within about 47 '. 4 (1.84 pc) of Sgr A* of at least $7.5 \times$ $10^{6} M_{\odot}$, assuming Keplerian motion in an edge-on disk at $8 \mathrm{kpc}$ distance. Granting up to $3.7 \times 10^{6} M_{\odot}$ for the black hole at the position of $\mathrm{Sgr}^{*}$ (Schödel et al. 2002), this would then imply that at least $50 \%$ of the total mass within the CND is contained in the enclosed stellar cluster and molecular cloud complexes. As expected, none of the $1720-\mathrm{MHz}$ (shock-excited) masers coincide with the circumstellar 1612$\mathrm{MHz}$ (FIR pumped) masers.

Four new 1667-MHz OH masers are presented in Table 2, all of them identified as circumstellar masers found by Lindqvist et al. (1992) in the 1612-MHz line. Two of them, corresponding to the strongest $1612-\mathrm{MHz}$ masers, are also newly detected in the $1665-\mathrm{MHz}$ line. Although we are dealing with (mostly) intrinsic variable sources, we can compare the data from the different transitions for each star because the data sets are taken simultaneously. Several interesting observations 


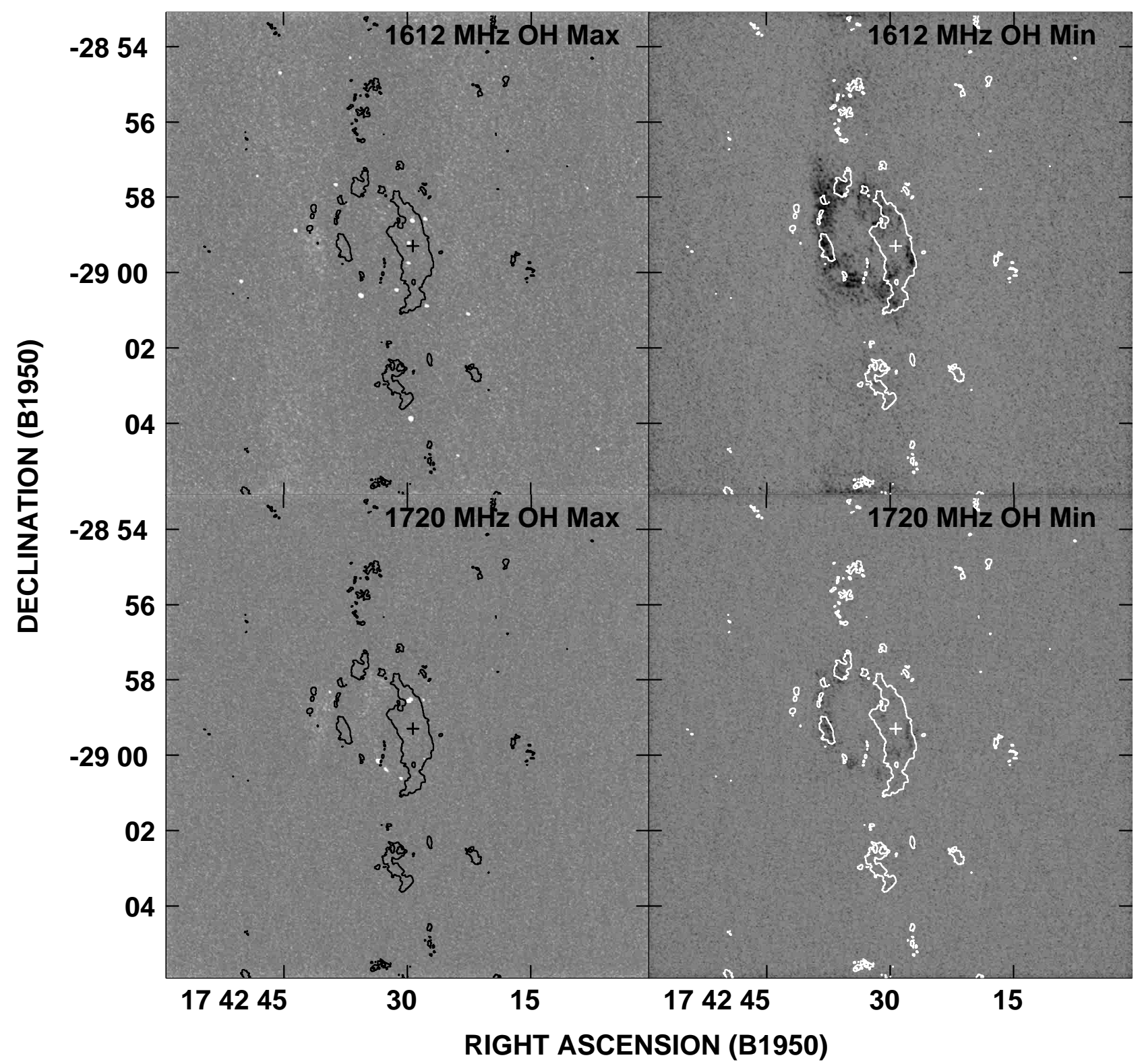

Fig. 9. 18-cm OH satellite line summary; Maxmaps and Minmaps at $1612 \mathrm{MHz}$ and $1720 \mathrm{MHz}$. Continuum contours and a cross at the position of $\mathrm{Sgr} \mathrm{A}^{*}$ are placed to guide the eye. The gray scale showing the relative noise of the maps is set to match that of the main lines (see text).

can already be made, although one should be cautious with the small numbers involved.

First, the 1665-MHz masers are only found in the strongest $1612-\mathrm{MHz}$ masers at a level of 1.5 and $2.8 \%$ with respect to the $1612-\mathrm{MHz}$ flux. If this $\sim 2 \%$ is a characteristic fraction, and recalling that the noise in the maps is about $5 \mathrm{mJy} / \mathrm{beam}$, then indeed these two sources are the only ones that would exceed five times the noise level at $1665-\mathrm{MHz}$.

Second, the two sources that show $1665-\mathrm{MHz}$ masers (i.e. the strongest 1612-MHz sources OH359.880-0.087 and OH359.938-0.078) also show 1667-MHz maser emission at a level between 4.4 and $9.5 \%$ of the $1612-\mathrm{MHz}$ flux. Inspecting the rotational transition levels of $\mathrm{OH}$, it becomes clear that in $\mathrm{OH} 359.952-0.036$ and $\mathrm{OH} 359.977-0.087$, the $1612-\mathrm{MHz}$ and $1667-\mathrm{MHz}$ masers are competing to populate the $J=3 / 2$, negative parity $F=2$ level. That $\mathrm{OH} 359.880-0.087$ and
OH359.938-0.078 lack in relative 1667:1612-MHz intensity might be due to the influence of the $1665-\mathrm{MHz}$ line, where the relative 1667:1612-MHz intensity in particular seems to decrease with the stronger $1665-\mathrm{MHz}$ maser.

As a final remark, although $\mathrm{OH} 359.952-0.036$ and OH359.977-0.087 are "normal" 1612-MHz maser emitters, none of the other "normal" $1612-\mathrm{MHz}$ masers that would have about $13 \%$ of the $1612-\mathrm{MHz}$ intensity above the noise (25 mJy/beam) has been found in the 1667-MHz line. Except for a very weak (18.67 mag) $K$-band $2.2 \mu \mathrm{m}$ counterpart for OH359.880-0.087 measured by Blommaert et al. (1998), none of the $1667-\mathrm{MHz}$ masing stars have been found in the $K$-band by either Blommaert et al. (1998), nor by Glass et al. (2001). In that respect the $1667-\mathrm{MHz}$ masers are not normal - they are "redder" than the others. It should also be noted that $\mathrm{OH} 359.938-0.078$ and $\mathrm{OH} 359.952-0.036$ are not variable in 
Table 2. Point source detections.

\begin{tabular}{|c|c|c|c|c|c|c|c|}
\hline \multirow[t]{2}{*}{ Name } & \multicolumn{2}{|c|}{ Position in B1950 } & \multicolumn{2}{|c|}{ Position in $\mathrm{J} 2000$} & \multirow{2}{*}{$\begin{array}{r}v_{\text {system }} \\
\left(\mathrm{km} \mathrm{s}^{-1}\right)\end{array}$} & \multirow{2}{*}{$\begin{array}{r}\text { Flux } \\
(\mathrm{mJy})\end{array}$} & \multirow[t]{2}{*}{ Reference } \\
\hline & (RA) & $(\mathrm{Dec})$ & (RA) & $(\mathrm{Dec})$ & & & \\
\hline \multicolumn{8}{|c|}{ OH 1612 MHz masers } \\
\hline ОН359.825-0.024 & 174206.961 & -290440.90 & 174517.824 & -290552.10 & -56 & 190 & L-92 (47) \\
\hline OH359.924+0.034 & 174208.300 & -285744.95 & 174518.987 & -285856.05 & -83 & 72 & L-92 (60) \\
\hline OH359.990+0.030 & 174218.210 & -285432.29 & 174528.817 & -285542.66 & -65 & 64 & S-98 \\
\hline OH359.906-0.036 & 174221.493 & -290054.62 & 174532.262 & -290204.73 & -47 & 40 & S-98 \\
\hline OH359.888-0.051 & 174222.486 & -290219.15 & 174533.292 & -290329.21 & -56 & 62 & L-92 (53) \\
\hline OH359.906-0.041 & 174222.714 & -290105.75 & 174533.488 & -290215.76 & -138 & 284 & L-92 (58) \\
\hline OH359.855-0.078 & 174223.935 & -290451.52 & 174534.805 & -290601.43 & +3 & 129 & L-92 (50) \\
\hline OH359.911-0.059 & 174227.482 & -290122.96 & 174538.264 & -290232.61 & -79 & 54 & L-92 (59) \\
\hline OH359.918-0.055 & 174227.700 & -290053.27 & 174538.469 & -290202.89 & -292 & 299 & S-98 \\
\hline OH359.952-0.036 & 174227.897 & -285835.23 & 174538.608 & -285944.83 & +85 & 289 & L-92 (66) \\
\hline OH359.954-0.041 & 174229.463 & -285837.61 & 174540.175 & -285947.09 & +71 & 731 & L-92 (67) \\
\hline OH359.880-0.087 & 174229.641 & -290352.92 & 174540.486 & -290502.37 & -20 & 1085 & L-92 (52) \\
\hline OH359.946-0.047 & 174229.892 & -285913.47 & 174540.619 & -290022.89 & -24 & 145 & L-92 (65) \\
\hline OH359.939-0.052 & 174229.919 & -285945.56 & 174540.659 & -290054.97 & +53 & 256 & L-92 (63) \\
\hline OH359.932-0.059 & 174230.575 & -290016.96 & 174541.329 & -290126.38 & -156 & 44 & S-98 \\
\hline OH359.932-0.063 & 174231.383 & -290027.57 & 174542.141 & -290136.86 & -92 & 286 & L-92 (61) \\
\hline OH359.970-0.049 & 174233.681 & -285803.05 & 174544.381 & -285912.31 & +89 & 39 & L-92 (69) \\
\hline OH359.985-0.042 & 174234.034 & -285704.34 & 174544.707 & -285813.46 & -11 & 28 & S-98 \\
\hline OH359.938-0.077 & 174235.685 & -290036.57 & 174546.447 & -290145.54 & -83 & 2408 & L-92 (62) \\
\hline ОH359.902-0.103 & 174236.608 & -290313.52 & 174547.439 & -290422.56 & -102 & 44 & S-98 \\
\hline $\mathrm{OH} \quad 0.030-0.026$ & 174236.999 & -285417.89 & 174547.602 & -285526.76 & -56 & 70 & S-98 \\
\hline OH359.986-0.061 & 174238.742 & -285739.90 & 174549.430 & -285848.63 & +16 & 246 & L-92 (73) \\
\hline OH359.980-0.077 & 174241.720 & -285827.08 & 174552.428 & -285935.59 & +89 & 40 & S-98 \\
\hline OH359.977-0.087 & 174243.616 & -285853.52 & 174554.336 & -290001.88 & +12 & 220 & L-92 (72) \\
\hline $\mathrm{OH} \quad 0.040-0.056$ & 174245.477 & -285442.46 & 174556.091 & -285550.68 & +71 & 147 & L-92 (81) \\
\hline $\mathrm{OH} \quad 0.007-0.088$ & 174248.232 & -285726.00 & 174558.915 & -285834.00 & +3 & 76 & L-92 (77) \\
\hline $\mathrm{OH} \quad 0.053-0.062$ & 174248.853 & -285414.07 & 174559.458 & -285522.21 & +7 & 26 & L-92 (82) \\
\hline ОН359.971-0.119 & 174250.180 & -290014.39 & 174600.935 & -290122.24 & -11 & 227 & L-92 (70) \\
\hline OH359.936-0.144 & 174251.127 & -290249.22 & 174601.947 & -290356.99 & -11 & 61 & L-92 (7-s) \\
\hline \multicolumn{8}{|c|}{ OH 1665 MHz masers } \\
\hline ОH359.880-0.087 & 174229.628 & -290352.91 & 174540.472 & -290502.27 & -29 & 30 & \\
\hline OH359.938-0.077 & 174235.678 & -290036.25 & 174546.440 & -290145.16 & -91 & 35 & \\
\hline \multicolumn{8}{|c|}{ OH 1667 MHz masers } \\
\hline OH359.952-0.036 & 174227.907 & -285835.21 & 174538.618 & -285944.75 & +63 & 37 & \\
\hline OH359.880-0.087 & 174229.597 & -290353.40 & 174540.442 & -290502.80 & -29 & 103 & \\
\hline OH359.938-0.077 & 174235.682 & -290036.55 & 174546.444 & -290145.50 & -82 & 106 & \\
\hline OH359.977-0.087 & 174243.647 & -285853.57 & 174554.367 & -290001.94 & -3 & 29 & \\
\hline \multicolumn{8}{|c|}{ OH 1720 MHz masers } \\
\hline ОН359.930-0.049 & 174228.017 & -290005.83 & 174538.764 & -290115.27 & -132 & 70 & YZ01 \\
\hline OH359.952-0.036 & 174228.181 & -285831.47 & 174538.888 & -285940.89 & +47 & 118 & YZ96 (C) \\
\hline OH359.955-0.040 & 174229.666 & -285832.18 & 174540.374 & -285941.48 & +132 & 290 & YZ96 (B) \\
\hline OH359.960-0.037 & 174229.705 & -285809.87 & 174540.403 & -285919.16 & +30 & 37 & \\
\hline OH359.955-0.042 & 174229.913 & -285834.44 & 174540.621 & -285943.71 & +132 & 658 & YZ96 (B) \\
\hline OH359.928-0.062 & 174230.843 & -290037.40 & 174541.603 & -290146.59 & +56 & 131 & YZ96 (G) \\
\hline OH359.934-0.066 & 174232.616 & -290023.40 & 174543.371 & -290132.45 & +56 & 248 & YZ96 (D,E,F) \\
\hline OH359.939-0.067 & 174233.588 & -290009.07 & 174544.337 & -290118.04 & +64 & 1290 & YZ96 (A) \\
\hline OH359.966-0.056 & 174234.878 & -285828.00 & 174545.586 & -285937.03 & +39 & 75 & \\
\hline OH359.977-0.069 & 174239.462 & -285819.67 & 174550.165 & -285928.20 & +56 & 93 & \\
\hline
\end{tabular}

their 1612-MHz maser emission, whereas OH359.977-0.087 has a, for the GC region, long period of 1070 days. The source with a $K$-band detection, OH359.880-0.087, has a more typical period of 760 days (the periods are taken from van Langevelde et al. 1993). It is tempting to conclude that these sources are evolving away from the OH/IR star stage to 
become planetary nebulae, where the non-variable $1612-\mathrm{MHz}$ sources $\mathrm{OH} 359.938-0.078$ and $\mathrm{OH} 359.952-0.036$ are the most transformed sources, and where OH359.880-0.087 has just started the transition.

\section{Summary}

We have performed observations of the four ground rotational state $\mathrm{OH}$ transitions towards the Galactic Centre with the VLA in the BnA configuration. Presented here are velocity maps of $\mathrm{OH}$ absorption at $9 \mathrm{~km} \mathrm{~s}^{-1}$ intervals and with angular resolution of $\approx 4^{\prime \prime}$. Maps of the $-T_{\mathrm{L}} / T_{\mathrm{C}}$ distribution for selected regions have confirmed and strengthened the reality of features and phenomena revealed in the absorption line maps. In a forthcoming paper, we shall determine relative intensities and optical depths of the structural features in all four ground-state lines, as well as perform a more detailed physical analysis of this large amount of data.

Acknowledgements. We are grateful to Jan Högbom for sharing his insight into the wonders of interferometry and CLEAN with us. Frank Gardner participated in the early stages of this project. This research was supported by the Swedish Research Council. LOS acknowledges support for this research by the European Commission under contract ERBFGECT950012 and HPRI-CT-1999-00045. The authors acknowledge the open policy for the use of NRAO's VLA. The National Radio Astronomy Observatory (NRAO) is operated by Associated Universities Inc., under cooperative agreement with the National Science Foundation.

\section{References}

Bieging, J. H. 1976, A\&A, 51, 289

Blommaert, J. A. D. L., van der Veen, W. E. C. J., van Langevelde, H. J., Habing, H. J., \& Sjouwerman, L. O. 1998, A\&A, 329, 991

Coil, A. L., \& Ho, P. T. P. 2000, ApJ, 533, 245

Cotera, A. S., Simpson, J. P., Erickson, E. F., et al. 2000, ApJS, 129, 123

Downes, D., \& Martin, H. M. 1971, Nature, 233, 112

Ekers, R. D., van Gorkom, J. H., Schwarz, U. J., \& Goss, W. M. 1983, A\&A, 122, 143

Genzel, R. 1989, The Circum-Nuclear Disk, in The Center of the Galaxy, ed. M. Morris (Dordrecht: Kluwer), IAU Symp., 136, 89

Glass, I. S., Matsumoto, S., Carter, B. S., \& Sekiguchi, K. 2001, MNRAS, 321, 77

Goss, W. M., Schwarz, U. J., van Gorkom, J. H., \& Ekers, R. D. 1985, MNRAS, 215, 69P

Güsten, R. 1989, Gas and Dust in the Inner Few Degrees of the Galaxy, in The Center of the Galaxy, ed. M. Morris (Dordrecht: Kluwer), IAU Symp., 136, 89
Habing, H. J., Olnon, F. M., Winnberg, A., Matthews, H. E., \& Baud, B. 1983, A\&A, 128, 230

Killeen, N. E. B., Lo, K. Y., \& Crutcher, R. 1992, ApJ, 385, 585

Lasenby, J., Lasenby, A. N., \& Yusef-Zadeh, F. 1989, ApJ, 343, 177

Lindqvist, M., Winnberg, A., Habing, H. J., \& Matthew, H. E. 1992, A\&AS, 92, 43 (L-92)

Lindqvist, M., Sandqvist, Aa., Winnberg, A., Johansson, L. E. B., \& Nyman, L.-Å. 1995, A\&AS, 113, 257

Liszt, H. S., van der Hulst, J. M., Burton, W. B., \& Ondrechen, M. P. 1983, A\&A, 126, 341

Mezger, P. G., Duschl, W. J., \& Zylka, R. 1996, A\&ARv, 7, 289

Morris, M., \& Serabyn, E. 1996, ARA\&A, 34, 645

Pauls, T., Johnston, K. J., \& Wilson, T. L. 1996, ApJ, 461, 223

Pedlar, A., Anantharamaiah, K. R., Ekers, R. D., et al. 1989, ApJ, 342, 769

Pihlström, Y. M., Conway, J. E., Booth, R. S., Diamond, P. J., \& Polatidis, A. G. 2001, A\&A, 377, 413

Plante, R. L., Lo, K. Y., \& Crutcher, R. M. 1995, ApJ, 445, L113

Sandqvist, Aa. 1971, Lunar Occultations of the Galactic Center Region in $\mathrm{HI}, \mathrm{OH}$ and $\mathrm{CH}_{2} \mathrm{O}$ Lines, Ph.D. Thesis (University of Maryland)

Sandqvist, Aa. 1973, A\&AS, 9, 391

Sandqvist, Aa. 1974, A\&A, 33, 413

Sandqvist, Aa. 1989, A\&A, 223, 293

Sandqvist, Aa., Karlsson, R., Whiteoak, J. B., \& Gardner, F. F. 1987, The 18-cm OH Distribution in the Galactic Center Torus, in The Galactic Center, ed. D. C. Backer (New York: AIP), AIP Conf. Proc., 155, 95

Sandqvist, Aa., Karlsson, R., \& Whiteoak, J. B. 1989, OH in the Environment of Sgr A, in The Center of the Galaxy, ed. M. Morris (Dordrecht: Kluwer), IAU Symp., 136, 421

Schödel, R., Ott, T., Genzel, R., et al. 2002, Nature, 419, 694

Sjouwerman, L. O. 1997, The OH/IR star population in the Galactic center, Ph.D. Thesis, Technical Report No. 316 (Chalmers University of Technology)

Sjouwerman, L. O., van Langevelde, H. J., Winnberg, A., \& Habing, H. J. 1998, A\&AS, 128, 35 (S-98)

van Langevelde, H. J., Janssens, A. M., Goss, W. M., Habing, H. J., \& Winnberg, A. 1993, A\&AS, 101, 109

Whiteoak, J. B., \& Gardner, F. F. 1976, MNRAS, 174, 627

Whiteoak, J. B., Rogstad, D. H., \& Lockhart, I. A. 1974, A\&A, 36, 245

Whiteoak, J. B., Gardner, F. F., \& Pankonin, V. 1983, MNRAS, 202, $11 \mathrm{P}$

Yusef-Zadeh, F., Lasenby, A., \& Marshall, J. 1993, ApJ, 410, L27

Yusef-Zadeh, F., Roberts, D. A., Goss, W. M., Frail, D. A., \& Green, A. J. 1996, ApJ, 466, L25 (YZ96)

Yusef-Zadeh, F., Goss, W. M., Roberts, D. A., Robinson, B., \& Frail, D. A. 1999, ApJ, 527, 172

Yusef-Zadeh, F., Roberts, D. A., Bower, G., Wardle, M., \& Goss, W. M. 2001, in Cosmic Masers, ed. V. Migenes, IAU Symp., 206, preprint (YZ01)

Zhao, J.-H., Goss, W. M., \& Ho, P. T. P. 1995, ApJ, 450, 122

Zylka, R., Mezger, P. G., \& Wink, J. E. 1990, A\&A, 234, 133 